AQ2

\title{
Complexation-mediated
}

\section{electromembrane extraction of highly polar basic drugs - a fundamental study with catecholamines in urine as model system}

AQ1

Elena Fernández, 1

Linda Vårdal, 2

Lorena Vidal, $1 \bowtie$

Email lorena.vidal@ua.es

Antonio Canals, 1

Astrid Gjelstad, 2

Stig Pedersen-Bjergaard, 2凶

Email stig.pedersen-bjergaard@farmasi.uio.no

1 Department of Analytical Chemistry, Nutrition and Food Sciences and University Institute of Materials, University of Alicante, P.O. Box 99, 03080 Alicante, Spain

2 School of Pharmacy, University of Oslo, P.O. Box 1068, Blindern, 0316 Oslo, Norway

\section{Abstract}

Complexation-mediated electromembrane extraction (EME) of highly polar basic drugs $(\log P<-1)$ was investigated for the first time with the catecholamines epinephrine, norepinephrine, and dopamine as model 
analytes. The model analytes were extracted as cationic species from urine samples ( $\mathrm{pH} 4$ ), through a supported liquid membrane (SLM) comprising $25 \mathrm{mM} \mathrm{4-(trifluoromethyl)phenylboronic} \mathrm{acid} \mathrm{(TFPBA)} \mathrm{in}$ bis(2-ethylhexyl) phosphite (DEHPi), and into $20 \mathrm{mM}$ formic acid as acceptor solution. EME was performed for $15 \mathrm{~min}$, and $50 \mathrm{~V}$ was used as extraction voltage across the SLM. TFPBA served as complexation reagent, and selectively formed boronate esters by reversible covalent binding with the model analytes at the sample/SLM interface. This enhanced the mass transfer of the highly polar model analytes across the SLM, and EME of basic drugs with $\log P$ in the range -1 to -2 was shown for the first time. Meanwhile, most matrix components in urine were unable to pass the SLM. Thus, the proposed concept provided highly efficient sample clean-up and the system current across the SLM was kept below $50 \mu \mathrm{A}$. Finally, the complexation-mediated EME concept was combined with ultra-high performance liquid chromatography coupled to tandem mass spectrometry and evaluated for quantification of epinephrine and dopamine. Standard addition calibration was applied to a pooled human urine sample. Calibration curves using standards between 25 and $125 \mathrm{~g} \mathrm{~L} \mathrm{~L}^{-1}$ gave a high level of linearity with a correlation coefficient of 0.990 for epinephrine and 0.996 for dopamine $(N=5)$. The limit of detection, calculated as three times signal-to-noise ratio, was $5.0 \mu \mathrm{g} \mathrm{L}^{-1}$ for epinephrine and $1.8 \mu \mathrm{g} \mathrm{L}^{-1}$ for dopamine. The repeatability of the method, expressed as coefficient of variation, was $13 \%(n=5)$. The proposed method was finally applied for the analysis of spiked pooled human urine sample, obtaining relative recoveries of 91 and $117 \%$ for epinephrine and dopamine, respectively.

\section{Keywords}

Electromembrane extraction

Polar analytes

Urine samples

Catecholamines

\section{Electronic supplementary material}

The online version of this article (doi: 10.1007/s00216-017-0370-2 ) contains supplementary material, which is available to authorized users. 


\section{Introduction}

Electromembrane extraction (EME) is a miniaturized extraction technique evolved from hollow fiber liquid-phase microextraction (HF-LPME) [1]. In EME, charged analytes are extracted from aqueous sample, through an organic solvent immobilized as a supported liquid membrane (SLM) in the pores of a polymeric hollow fiber, and into an acceptor solution located in the lumen of the fiber [2]. An electrical potential difference is employed as driving force for the electrokinetic migration of analytes across the SLM. A power supply provides a DC potential between two electrodes placed in the sample and acceptor solution, respectively. For the extraction of basic analytes, the anode (positively charged electrode) is placed into sample whereas the cathode (negatively charge electrode) is placed into the acceptor solution. For the extraction of acidic analytes, the direction of the electrical field is reversed, the cathode is located in the sample and the anode is located in the acceptor solution. The $\mathrm{pH}$ of both sample and acceptor solution has to be controlled to ensure full ionization of the target analytes. Major advantages of EME include the following[3, 4]: low consumption of organic solvents; shorter extraction times than HF-LPME due to the enhancement of mass transport by the force of the electrical potential; efficient sample clean-up and feasibility of direct extraction from untreated complex matrices; easy extraction selectivity modulation by changes in the magnitude and direction of the electrical potential; high preconcentration capacity; direct compatibility with a wide range of analytical instruments; simple and low cost equipment; and possibilities of downscaled format (i.e., microchip devices) and automation.

Experimental parameters such as the SLM composition, extraction voltage, extraction time, $\mathrm{pH}$ of sample and acceptor solutions, salt effect, and sample stirring speed strongly affect EME performance, and are normally optimized in different applications [2,3]. The selection of appropriate solvent within the pores of the fiber is a critical task of the technique. Some important properties of the solvent to consider are immiscibility with water to prevent losses by dissolution, low volatility to avoid evaporation during extraction, low viscosity to ensure high diffusion coefficients across SLM, good extractability and high partition coefficient of the target analytes, and certain dipole moment or conductivity to support current flow in the system $[3,5]$. For the EME of non-polar $(\log P>2)$ basic drugs, 2-nitrophenyl octylether (NPOE) $[6,7,8,9,10,11,12,13,14]$ has 
been the most employed solvent, although 1-ethyl-2-nitrobenzene (ENB) $[15,16,17,18]$ and 1-isopropyl-4-nitrobenzene (IPNB) $[19,20]$ have been alternatively proposed, performing extractions at low voltages. NPOE, ENB, and IPNB possess low water solubility, high boiling point, and are able to form dipole-dipole and hydrogen bonding interactions with positively charged analytes, thus being suitable solvents to create efficient SLMs [20, 21]. The extraction of polar $(\log P<2)$ basic drugs is more challenging since these species are less prone to cross the hydrophobic SLM under the influence of an electrical field. In this case, the presence of carriers in the SLM is compulsory to promote the analyte transfer and to increase EME efficiency. Among tested carriers, di(2-ethylhexyl) phosphate (DEHP) and tri(2-ethylhexyl) phosphate (TEHP) have been the most popular ones [21]. DEHP forms ion-pairs with positively charged basic drugs, whereas TEHP is a non-ionic carrier interacting with charged analytes mainly by dipole-dipole and hydrogen interactions. DEHP has been more efficient than TEHP for the extraction of the most polar basic drugs $(0.01<\log P<1.8)$ [21]. However, DEHP suffers from some drawbacks related to the increase of the electrical conductance of the SLM and extraction of background electrolyte ions and other ionic sample components, leading to high system currents [22]. Very recently, a new SLM based on bis(2-ethylhexyl) phosphite (DEHPi) has been discovered as a good candidate for the extraction of polar $(\log P$ values between -0.40 and 1.32) basic analytes from plasma samples [22]. DEHPi was compared with SLMs based on DEHP and TEHP, and DEHPi provided lower currents and higher system stability [22].

Experiences with EME of basic drug substances of very high polarity $(-1>\log P>-2)$ have not yet been reported in the literature, and therefore a fundamental study on this was addressed in the present work. The catecholamines dopamine (DA) (log $P=-0.99)$, epinephrine (E) $(\log$ $P=-1.37)$, and norepinephrine (NE) $(\log P=-1.85)$ were selected as model analytes [23]. In order to enhance their mass transfer across the SLM, and to maintain an acceptable level of selectivity and sample clean-up from biological fluids, different analogues of phenylboronic acid (PBA) were added to the EME system as selective complexation reagents for the catecholamines. Operational parameters for this conceptually new type of complexation-mediated EME system were studied and optimized to obtain fundamental experience and knowledge. Special emphasis was 
devoted to recovery, current stability, and sample clean-up. The optimized EME system was finally combined with ultra-high performance liquid chromatography coupled to tandem mass spectrometry (UHPLC-MS/MS), and evaluated for the quantification of DA and $\mathrm{E}$ in human urine.

\section{Experimental part}

\section{Chemicals}

Dopamine hydrochloride, epinephrine hydrochloride, norepinephrine bitartrate, 1,4-benzodioxane-6-boronic acid, 4-(trifluoromethyl)phenylboronic acid (TFPBA), $m$-tolylboronic acid, 4-(benzyloxy)phenylboronic acid, 4-(dimethylamino)phenylboronic acid, 4-(trans-2-carboxyvinyl)phenylboronic acid, DEHP, DEHPi, formic acid, and sodium 1-heptanesulfonate were all purchased from Sigma-Aldrich (St. Louis, MO, USA). PBA and NPOE were obtained from Fluka (Buchs, Switzerland). Hydrochloric acid, phosphoric acid, sodium dihydrogen phosphate monohydrate, disodium hydrogen phosphate dodecahydrate, trisodium phosphate dodecahydrate, and methanol were supplied by Merck (Darmstadt, Germany). The ultrapure water (resistivity of $18.2 \mathrm{M} \Omega \mathrm{cm}$ at $25^{\circ} \mathrm{C}$ ) employed for preparing aqueous solutions was obtained with a Milli-Q water purification system (Molsheim, France).

\section{Solutions and urine samples}

Stock solutions of E, NE, and DA were prepared at $1000 \mathrm{mg} \mathrm{L}^{-1}$ in methanol and stored at $5{ }^{\circ} \mathrm{C}$ protected from light. Aqueous working solutions were daily prepared by proper dilution of stock solutions with selected background electrolyte (i.e., $10 \mathrm{mM}$ hydrochloric acid or $20 \mathrm{mM}$ phosphate buffer). Solutions of $1 \mathrm{mg} \mathrm{L}^{-1}$ containing the three analytes were employed in initial experiments and EME optimization.

Urine samples were collected from healthy volunteers in sterilized containers and kept at $5{ }^{\circ} \mathrm{C}$ before analysis. Urine samples were diluted with $20 \mathrm{mM}$ phosphate buffer of predetermined $\mathrm{pH}$ (volume ratio 1:1) before EME experiments.

\section{Instrumentation}

Two chromatographic systems were employed for EME optimization and 
method evaluation, respectively. For EME optimization, chromatographic analysis was performed by high performance liquid chromatography coupled to ultraviolet detection (HPLC-UV). The chromatographic system containing a degasser, a binary pump, and an autosampler (all of 1200 series) was from Agilent Technologies (Santa Clara, CA, USA). Gemini C18 column ( $150 \mathrm{~mm} \times 2 \mathrm{~mm}$ I.D, $5 \mu \mathrm{m}$ particle size) from Phenomenex (Torrance, CA, USA) was employed for separation. The injection volume was $10 \mu \mathrm{L}$. Analytes were eluted in gradient mode using mobile phases A and B. Mobile phase A consisted of $95 \%$ water phase $(20 \mathrm{mM}$ formic acid and $5 \mathrm{mM}$ sodium 1-heptanesulfonate in ultrapure water) and 5\% methanol. Mobile phase B consisted of 95\% methanol and 5\% water phase (20 $\mathrm{mM}$ formic acid and $5 \mathrm{mM}$ sodium 1-heptanesulfonate in ultrapure water). Elution program was as follows: mobile phase B was increased from 3 to $35 \%$ within 12 min. Then, mobile phase B was further increased to $80 \%$ in $0.5 \mathrm{~min}$ and this condition was kept for $3.5 \mathrm{~min}$. Finally, the mobile phase composition was returned to the starting conditions and held constant for $4 \mathrm{~min}$ before next injection. The total analysis time was $20 \mathrm{~min}$ with a flow rate of $0.4 \mathrm{~mL} \mathrm{~min}^{-1}$. The UV detector was set at $280 \mathrm{~nm}$.

Method evaluation was carried out using UHPLC-MS/MS. The chromatographic system comprised a Dionex UltiMate 3000 RS pump, autosampler, and column compartment followed by a LTQ XL linear ion trap mass spectrometer from Thermo Scientific (San Jose, CA, USA). Chromatographic separation was achieved on an Acquity UPLC $®$ HSS T3 column $(100 \mathrm{~mm} \times 2.1 \mathrm{~mm}$ I.D, $1.8 \mu \mathrm{m}$ particle size $)$ from Waters (Wexford, Ireland) kept at $40{ }^{\circ} \mathrm{C}$. The injection volume was $5 \mu \mathrm{L}$. Mobile phase A contained $95 \%$ water phase $(20 \mathrm{mM}$ formic acid in ultrapure water) and 5\% methanol. Mobile phase B contained 95\% methanol and 5\% water phase (20 mM formic acid in ultrapure water). The linear gradient elution was programmed from 1 to $80 \%$ of mobile phase B in $1.5 \mathrm{~min}$. Eighty percent of mobile phase B was kept for 1 min before changing back to the starting conditions for equilibration. The total analysis time was 5.5 min with a flow rate of $0.3 \mathrm{~mL} \mathrm{~min}{ }^{-1}$. MS/MS detection was acquired in the selected reaction monitoring (SRM) mode with electrospray ionization in the positive mode. Transitions $(\mathrm{m} / \mathrm{z}) 184 \rightarrow 166$ and $154 \rightarrow 137$ were monitored for $\mathrm{E}$ and DA, respectively, for quantitative purposes. NE was excluded from the method evaluation in this conceptual work (i.e., 
UHPLC-MS/MS) since its quantification in the concentration range of interest (i.e., $\mu \mathrm{g} \mathrm{L}^{-1}$ level) was not achieved. The source fragmentation energy was $35 \mathrm{~V}$ and the collision energy was $15 \%$ for E and $17 \%$ for DA.

\section{EME set-up and procedure}

The sample compartment was a 2-mL glass vial with screw cap from Supelco (Bellefonte, PA, USA). The hollow fiber used as the support for the organic solvent and for housing the acceptor solution was a PP Q3/2 polypropylene hollow fiber from Membrana (Wuppertal, Germany) with an internal diameter of $1.2 \mathrm{~mm}$, wall thickness of $200 \mu \mathrm{m}$, and pore size of $0.2 \mu \mathrm{m}$. A Thermomixer Comfort agitator from Eppendorf (Hamburg, Germany) was used to agitate the extraction unit during EME. Platinum wires with $0.5 \mathrm{~mm}$ of diameter were used as electrodes. The electric potential was generated by a DC power supply (model ES 0300-0.45) from Delta Electronika (Zierikzee, The Netherlands). Current was monitored during EME using an Agilent U1253B True Rms Oled multimeter.

EME was performed according to the following procedure: $1 \mathrm{~mL}$ of sample solution was placed into $2 \mathrm{~mL}$ glass vial. The polypropylene hollow fiber was cut in a $2.5-\mathrm{cm}$ piece whose lower end was sealed by mechanical pressure. The upper end was connected by heat to a $2.2-\mathrm{cm}$ length pipette tip (Finntip 200 Ext from Thermo Scientific) acting as guiding tube. The hollow fiber was dipped for $5 \mathrm{~s}$ in the organic solvent used as SLM and the excess of solvent was thereafter removed with a medical wipe. Via guiding tube, $25 \mu \mathrm{L}$ of acceptor solution was filled into the lumen of the hollow fiber with a microsyringe. Subsequently, the hollow fiber was inserted through the vial cap and introduced in the sample. Finally, the cathode was placed in the acceptor solution and the anode in the sample. The electrodes were connected to the power supply and the extraction unit was agitated at $900 \mathrm{rpm}$ for a predetermined time. After EME, acceptor solution was collected with a microsyringe for its final injection in the corresponding chromatographic system (i.e., HPLC-UV for optimization studies and UHPLC-MS/MS to evaluate the method).

\section{Calculations}

The EME recovery was calculated using the following equation: 


$$
\text { Recovery }(\%)=\frac{C_{\mathrm{a}} V_{\mathrm{a}}}{C_{\mathrm{s}} V_{\mathrm{s}}} \times 100
$$

where $C_{\mathrm{a}}$ is the final concentration of the analyte in the acceptor solution, $C_{\mathrm{s}}$ is the initial analyte concentration in the sample solution, $V_{\mathrm{a}}$ is the volume of the acceptor solution, and $V_{\mathrm{s}}$ is the volume of the sample.

\section{Results and discussion}

\section{Experiments based on conventional EME}

First, experiments were performed using pure NPOE as SLM. The catecholamines were dissolved in $10 \mathrm{mM}$ hydrochloric acid ( $\mathrm{pH} 2)$, and this solution served as sample. EME was operated at $300 \mathrm{~V}$. After 5 min of extraction, no analytes were detected by HPLC-UV in the acceptor solution. The catecholamines were then dissolved in $20 \mathrm{mM}$ phosphate buffer ( $\mathrm{pH}$ 5), and with this solution serving as sample, EME was repeated under equal conditions. However, also in this case, no extraction of the catecholamines was observed. The inefficiency of NPOE was expected. NPOE is well known to efficiently extract non-polar basic compounds (log $P>2$ ) by strong dipole and hydrogen bonding interactions. On the other hand, the extraction of polar analytes with low affinity to the SLM generally requires the use of hydrophobic ion-pair reagents, such as DEHP, acting as carriers [21].

DEHP has been frequently combined with NPOE for the extraction of polar substances, since its ability to form complexes with positively charged species facilitates their transfer into the SLM [21]. A SLM based on NPOE with $10 \%(w / w)$ of DEHP was tested for the catecholamines using an extraction voltage of $25 \mathrm{~V}$. Standard solutions of $\mathrm{pH} 2$ and $5(10 \mathrm{mM}$ HClhydrochloric acid and $20 \mathrm{mM}$ phosphate buffer, respectively) were subjected to EME for $5 \mathrm{~min}$. Surprisingly, the analytes were not found in the corresponding acceptor solutions, even not at trace level. Thus, the SLM comprising a mixture of DEHP and NPOE appeared to be insufficient for mass transfer of the highly polar catecholamines.

DEHPi has been recently demonstrated as SLM for extraction of polar basic drugs in the $\log P$ range from -0.40 to 1.32 [22]. DEHPi was also tested in the current work for catecholamines using an applied voltage of 
$50 \mathrm{~V}$. After 5 min of extraction from an aqueous standard solution of $\mathrm{pH} 2$ (10 $\mathrm{mM}$ HCthydrochloric acid), catecholamines were now detected in the acceptor solution. The extraction recoveries were $0.3 \%$ for $\mathrm{E}, 0.4 \%$ for $\mathrm{NE}$, and $0.8 \%$ for DA. The experiment was repeated with 10 min extraction time, and extraction recoveries increase to $0.5 \%$ for $\mathrm{E}, 0.7 \%$ for $\mathrm{NE}$, and $1.6 \%$ for DA. However, a more significant improvement was observed using a standard solution of pH 5 (20 mM phosphate buffer), and recoveries were now 3\% for E, $6 \%$ for $\mathrm{NE}$, and $14 \%$ for DA after $10 \mathrm{~min}$ of extraction. The $\mathrm{pH}$ dependence observed was unexpected since DEHPi is not able to form ionic interactions under normal $\mathrm{pH}$ conditions [22]. The enhancement in extraction performance at higher $\mathrm{pH}$ was hypothesized to be due to the presence of small amounts of ionic oxidation products in DEHPi. Thus, special attention should be paid in the manipulation of DEHPi, using closed containers to avoid its progressive oxidation as far as possible.

\section{Experiments based on complexation-mediated EME}

The molecular structures of the catecholamines include two phenolic groups in ortho position as a common feature. PBA and derivatives possess a high affinity to complex these phenols, forming boronate esters by reversible covalent binding (Fig. 1). Based on this type of complexation, previous publications [24, 25, 26] have reported the ability of PBA derivatives to facilitate transport of diol containing species (e.g., DA, glucoside, fructose) through SLMs under passive diffusion conditions. This concept was transferred to EME in the present work, and tested under electrokinetic migration conditions. The idea was to enhance the mass transfer of catecholamines due to selective complexation, while suppressing the general mass transfer of cationic matrix components.

\section{Fig. 1}

PBA complexation of diol groups

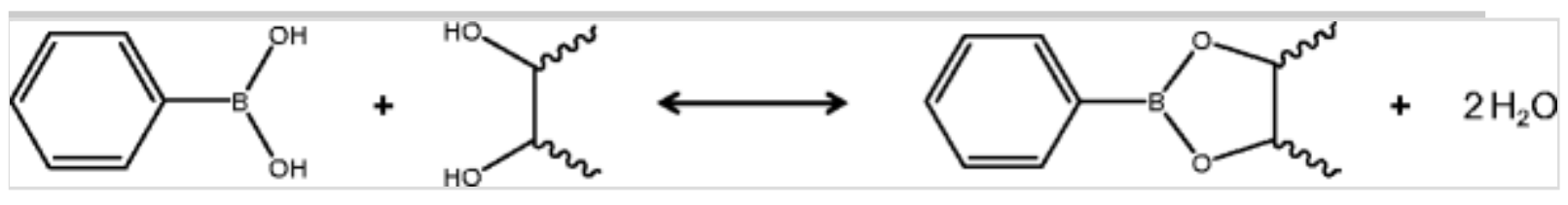

In a first experiment, PBA was dissolved in standard solution of $\mathrm{pH} 5$ at a concentration of $5 \mathrm{mM}$. EME was performed for $10 \mathrm{~min}$ at $50 \mathrm{~V}$ using 
DEHPi as SLM. Under these conditions, recoveries were 4\% for E, $10 \%$ for NE, and $20 \%$ for DA. The improvement in extraction efficiency, especially for NE and DA, was attributed to decreased polarity of these molecules via complexation (Fig. 1). Based on this positive finding, the potential for complexation-mediated EME was studied in more detail below.

\section{Optimization}

\section{Type of complexing reagent}

PBA and six different derivatives (namely 1,4-benzodioxane-6-boronic acid; TFPBA; $m$-tolylboronic acid; 4-(benzyloxy)phenylboronic acid; 4-(dimethylamino) phenylboronic acid; and 4-(trans2-carboxyvinyl)phenylboronic acid) were investigated using DEHPi as SLM. For stepwise development of experiences, optimization was performed with aqueous standard solutions. The complexing reagents were dissolved in the sample solution or in DEHPi depending on their polarity and water miscibility. Thus, PBA $(\log P=1.64)$ and 1,4-benzodioxane6-boronic acid $(\log P=0.95)$ were added to the aqueous sample, and with these reagents complexation was expected in the bulk sample. In contrast, TFPBA $(\log P=2.52), m$-tolylboronic acid $(\log P=2.11)$, and 4-(benzyloxy)-phenylboronic acid $(\log P=3.16)$ were dissolved in the SLM. With these reagents, complexation was expected at the sample/SLM interface. The use of equal amounts (moles) of the different reagents was considered necessary in order to compare their net effect on EME. Therefore, reagents in the aqueous standard $(1 \mathrm{~mL})$ were dissolved at a concentration of $1 \mathrm{mM}$, whereas reagents in the SLM (approximately $20 \mu \mathrm{L}$ ) were dissolved at a concentration of $50 \mathrm{mM}$. The dissolution of 4-(dimethylamino)phenylboronic acid $(\log P=1.90)$ and 4-(trans2-carboxyvinyl)phenylboronic acid $(\log P=1.99)$ in aqueous phase or DEHPi was not achieved at selected concentrations, and these derivatives were therefore discarded. The effect of the different complexing reagents on EME of catecholamines is shown in Fig. 2. As observed, higher recoveries were obtained with TFPBA dissolved in DEHPi, and therefore this reagent was selected for further investigations together with PBA. TFPBA and PBA were both tested with NPOE as SLM, but these EME systems were not efficient. Thus, DEHPi was used as SLM in all remaining experiments. 


\section{Fig. 2}

Effect of complexing reagent. Extraction conditions: concentration of analytes, $1 \mathrm{mg} \mathrm{L}^{-1}$; sample $\mathrm{pH}, 5$; SLM, DEHPi; applied voltage, $50 \mathrm{~V}$; extraction time, $10 \mathrm{~min}$; acceptor solution, $20 \mathrm{mM}$ formic acid. Error bars represent the standard deviation of three replicated analysis

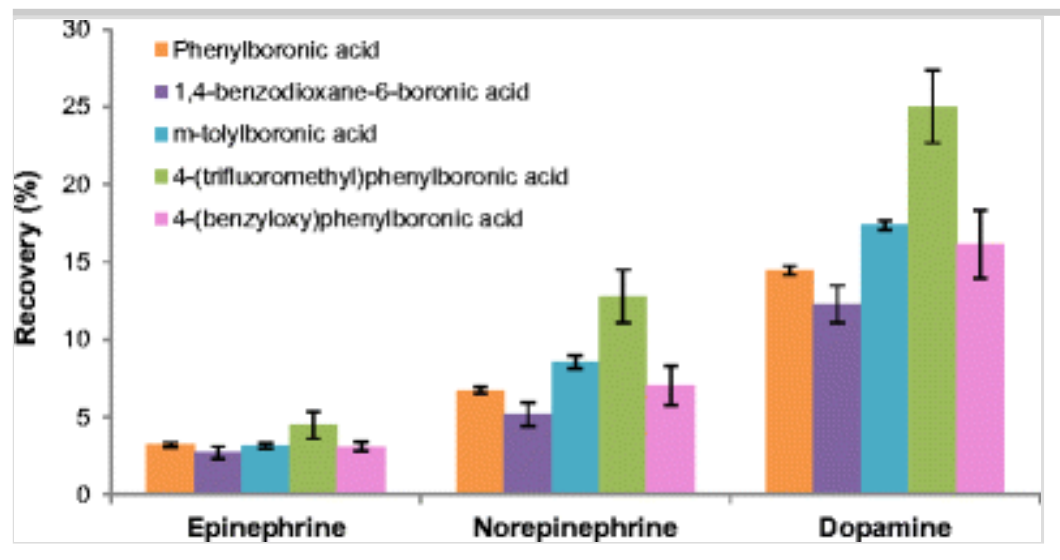

\section{Concentration of complexing reagent}

Different concentrations of PBA in the sample solution (i.e., 0, 0.5, 1, 3, 5, and $10 \mathrm{mM}$ ) and TFPBA in SLM (i.e., 0, 25, 50, and $250 \mathrm{mM}$ ) were evaluated. As shown in Fig. 3a, the effect of PBA on EME performance was practically negligible at concentrations of 0.5 and $1 \mathrm{mM}$. In these experiments, the molar concentration of PBA was 30-60 times higher than the analyte concentrations used in the experiment $\left(1 \mathrm{mg} \mathrm{L}^{-1}\right)$. However, a significant increase in extraction was observed at 3, 5, and $10 \mathrm{mM}$, especially for NE and DA. System current measurements revealed that current increased with PBA concentration, although it was kept below $50 \mu \mathrm{A}$ in all cases [22]. Finally, $3 \mathrm{mM}$ PBA was selected as optimum value since extraction recoveries were comparable to those obtained at higher concentrations (i.e., 5 and $10 \mathrm{mM}$ ), but the EME system was more stable. Regarding TFPBA, Fig. $3 \mathrm{~b}$ shows an enhancement in extraction performance as the reagent concentration increased. However, as observed with PBA, the system current increased with the concentration of the complexing reagent exceeding $50 \mu \mathrm{A}$ at $250 \mathrm{mM}$. Finally, $25 \mathrm{mM}$ TFPBA was selected as a compromise value.

\section{Fig. 3}

Effect of a PBA concentration, and $\mathbf{b}$ TFPBA concentration. Extraction conditions: concentration of analytes, $1 \mathrm{mg} \mathrm{L}^{-1}$; sample $\mathrm{pH}, 5$; SLM, 
DEHPi; applied voltage, $50 \mathrm{~V}$; extraction time, $10 \mathrm{~min}$; acceptor solution, $20 \mathrm{mM}$ formic acid. Error bars represent the standard deviation of three replicated analysis

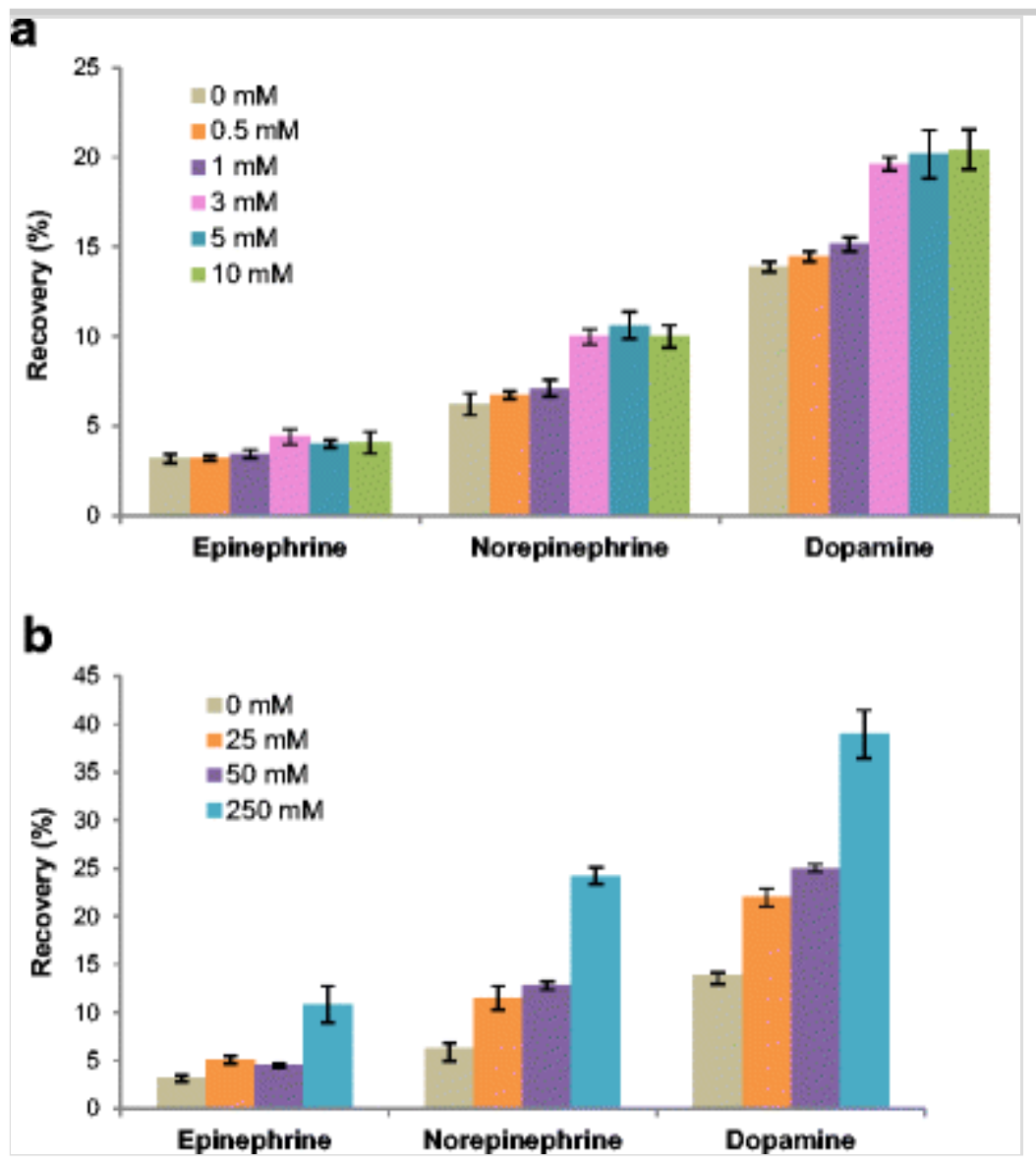

The EME of catecholamines using simultaneously $3 \mathrm{mM}$ PBA dissolved in sample solution (i.e., aqueous standard) and $25 \mathrm{mM}$ TFPBA dissolved in DEHPi was also tested. Recoveries were not significantly different to those obtained with complexing reagents separately and system current increased. Therefore, the simultaneous use of PBA and TFPBA was discarded.

Finally, selected optimum conditions (i.e., $3 \mathrm{mM}$ PBA or $25 \mathrm{mM}$ TFPBA dissolved in sample solution or DEHPi, respectively) were evaluated in a real urine sample diluted with $20 \mathrm{mM}$ phosphate buffer $\mathrm{pH} 5$ (volume ratio 1:1). Higher recoveries were obtained with $25 \mathrm{mM}$ TFPBA (see Electronic Supplementary Material (ESM) Fig. S1). Additionally, a general increase in system current was observed when EME was conducted from the real samples compared to aqueous standards. However, the increase in current was lower and kept below $50 \mu \mathrm{A}$ for TFPBA. According to these results, TFPBA was finally selected as complexing reagent for EME of the 
catecholamines.

Sample pH and acceptor solution composition

The effect of sample $\mathrm{pH}$ on EME was investigated in the range of $\mathrm{pH} 3-8$ using $20 \mathrm{mM}$ phosphate buffer solutions. As shown in Fig. S2 (see ESM), extraction recoveries were lower at $\mathrm{pH} 3$ and 8 whereas comparable values were obtained for $\mathrm{pH} 4,5,6$, and 7 . The drop in extraction efficiency at pH 3 could be related to a reduced affinity of TFPBA to complex target analytes under strongly acidic conditions. The drop in extraction efficiency at $\mathrm{pH} 8$ could be due to a partial negative ionization of the target analytes and, most likely, to the formation of anionic complexes with TFPBA [27]. Boronic acids can form neutral esters in non-polar solvents whereas they tend to form anionic boronate esters in water at basic $\mathrm{pH}$ [27]. At $\mathrm{pH} 8$, the formation of anionic complexes could be favored over the formation of neutral complexes. The transport of these negatively charged molecules through the SLM was hindered by the direction of the applied voltage, and thus, the extraction efficiency decreased.

Finally, $\mathrm{pH} 4$ was selected as optimum value in terms of recoveries, and also considering the higher stability of the target analytes under acidic conditions [23].

The effect of acceptor solution composition on complexation-mediated EME was evaluated employing acidic conditions to maintain the positive ionization of catecholamines. To this end, solutions of $20 \mathrm{mM}$ formic acid $(\mathrm{pH}=2.7), 200 \mathrm{mM}$ formic acid $(\mathrm{pH}=2.2), 20 \mathrm{mM}$ phosphate buffer $(\mathrm{pH}=2)$, and $10 \mathrm{mM}$ hydrochloric acid $(\mathrm{pH}=2)$ were prepared and used as acceptor solution in different experiments. EME was performed from pH 4 sample solution, using DEHPi with $25 \mathrm{mM}$ TFPBA in the SLM and an applied voltage of $50 \mathrm{~V}$. After $10 \mathrm{~min}$ of extraction, comparable extraction efficiencies (data not shown) were obtained with the different experiments, showing a negligible effect of the acceptor solution composition on the EME. Finally, $20 \mathrm{mM}$ formic acid was selected in subsequent experiments considering its compatibility with the UHPLC-MS/MS system use to evaluate the method.

Applied voltage and extraction time

The influence of applied voltage was studied from 0 to $100 \mathrm{~V}$. In 
experiments at $0 \mathrm{~V}$, catecholamines were not found in acceptor solution, and this supported that there were no passive diffusion of the model analytes in the current complexation-mediated EME systems. Thus, the use of voltage across the SLM was required to extract highly polar target analytes. The effect of voltage on complexation-mediated EME is shown in Fig. 4a. As expected, the extraction recoveries increased with increasing voltage up to $100 \mathrm{~V}$. However, system current also increased with the applied voltage, and the current exceeded $50 \mu \mathrm{A}$ at 75 and $100 \mathrm{~V}$. For urine samples, system current was also expected to exceed $50 \mu \mathrm{A}$ at 75 and $100 \mathrm{~V}$ and it was checked to be under this value at $50 \mathrm{~V}$. Finally, $50 \mathrm{~V}$ was chosen as optimum extraction voltage, compromising extraction recovery and EME system stability (current below $50 \mu \mathrm{A}$ ).

\section{Fig. 4}

Effect of a applied voltage, and $\mathbf{b}$ extraction time. Extraction conditions: concentration of analytes, $1 \mathrm{mg} \mathrm{L}^{-1}$; sample $\mathrm{pH}$, 4; SLM, DEHPi with $25 \mathrm{mM}$ TFPBA; applied voltage, $50 \mathrm{~V}$ (if not indicated); extraction time, $10 \mathrm{~min}$ (if not indicated); acceptor solution, $20 \mathrm{mM}$ formic acid. Error bars represent the standard deviation of three replicated analysis

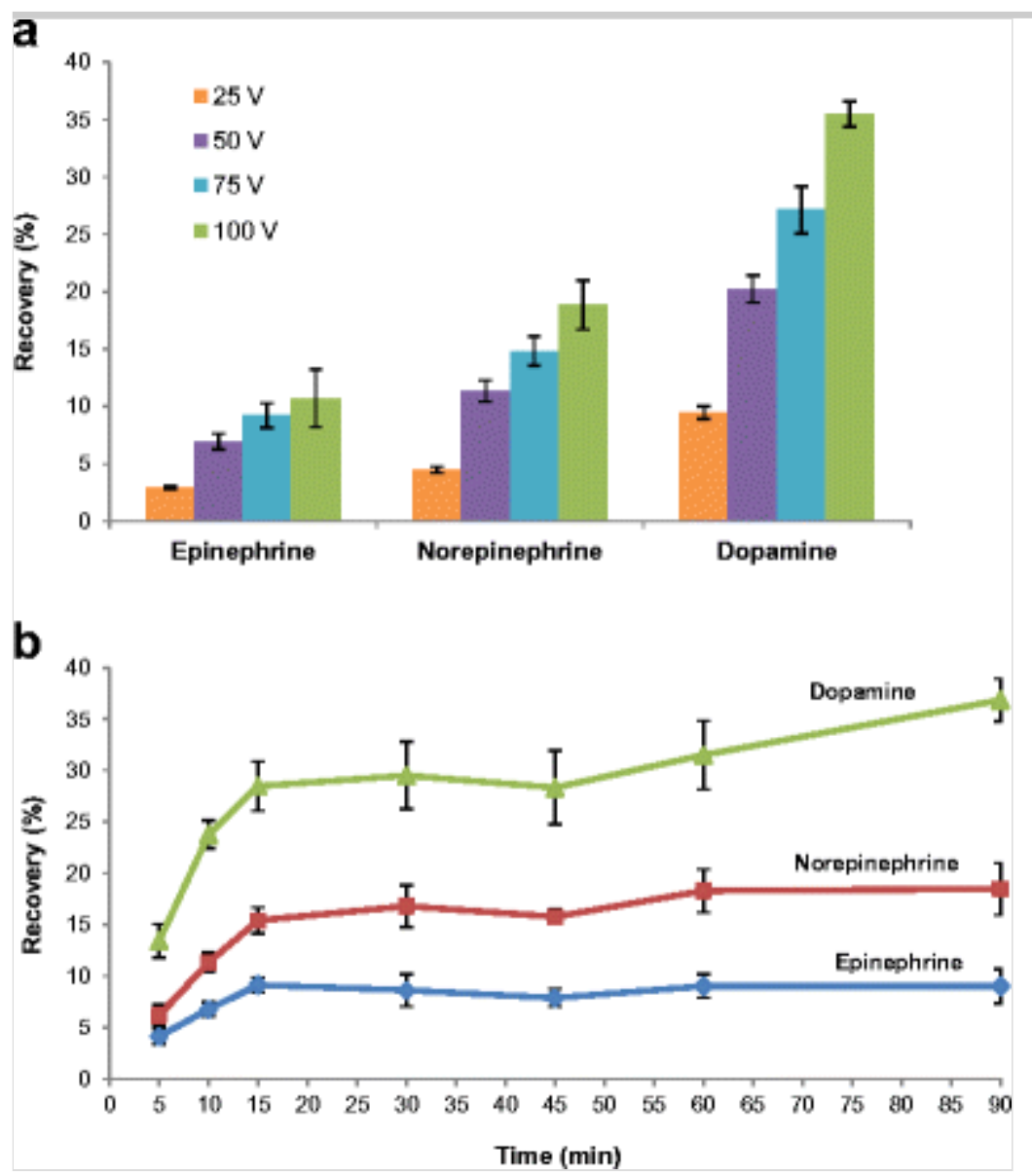


Finally, extraction time was investigated and the results are shown in Fig. 4b. Recoveries increased as a function of time during the first $15 \mathrm{~min}$ of extraction, as expected. Longer extraction times did not improve extraction recoveries and, according to previous publications, this effect could be attributed to $\mathrm{pH}$ changes in the acceptor solution due to electrolysis [22, $28]$. The extraction time effect was also evaluated in a real urine sample diluted with $20 \mathrm{mM}$ phosphate buffer $\mathrm{pH} 4$ (volume ratio 1:1). As with the aqueous samples, no improvement in recoveries was observed after $15 \mathrm{~min}$ of extraction (data not shown). Therefore, 15 min was finally selected as optimum time for complexation-mediated EME of the catecholamines.

\section{Extraction performance in urine samples under optimized conditions}

The final EME system was based on the following optimized conditions: SLM, DEHPi with $25 \mathrm{mM}$ TFPBA; sample $\mathrm{pH}$, 4; acceptor solution, $20 \mathrm{mM}$ formic acid; applied voltage, $50 \mathrm{~V}$; and extraction time, $15 \mathrm{~min}$. Under these conditions, recoveries were $10 \%$ for E, $15 \%$ for NE, and $29 \%$ for DA when EME was performed from aqueous standards. However, when analyzing urine samples, extraction recoveries decreased significantly as discussed in "Evaluation" section.

EME is known to provide excellent sample clean-up since the SLM forms a hydrophobic barrier between the sample and acceptor solution. Figure 5a shows HPLC-UV chromatograms before and after complexation-mediated EME of a diluted urine sample (volume ratio 1:1, urine/20 $\mathrm{mM}$ phosphate buffer $\mathrm{pH} 4$ ) at a $1 \mathrm{mg} \mathrm{L}^{-1}$ spiking level. Although some peaks from the sample matrix are present after EME, differences between the two chromatograms are obvious, indicating a high level of sample clean-up. This indicated that even though the complexation reagent improved the mass transfer of the highly polar model analytes across the SLM, the selective nature of this complexation prevented the bulk matrix of the urine sample from entering the SLM. Additionally, the system current profile is illustrated in Fig. 5b, showing that the complexation-mediated EME system was highly stable in contact with the diluted urine sample under optimized conditions.

\section{Fig. 5}

EME performance in urine sample under optimized conditions: a HPLC-UV 
chromatograms showing sample clean-up, and $\mathbf{b}$ system current profile

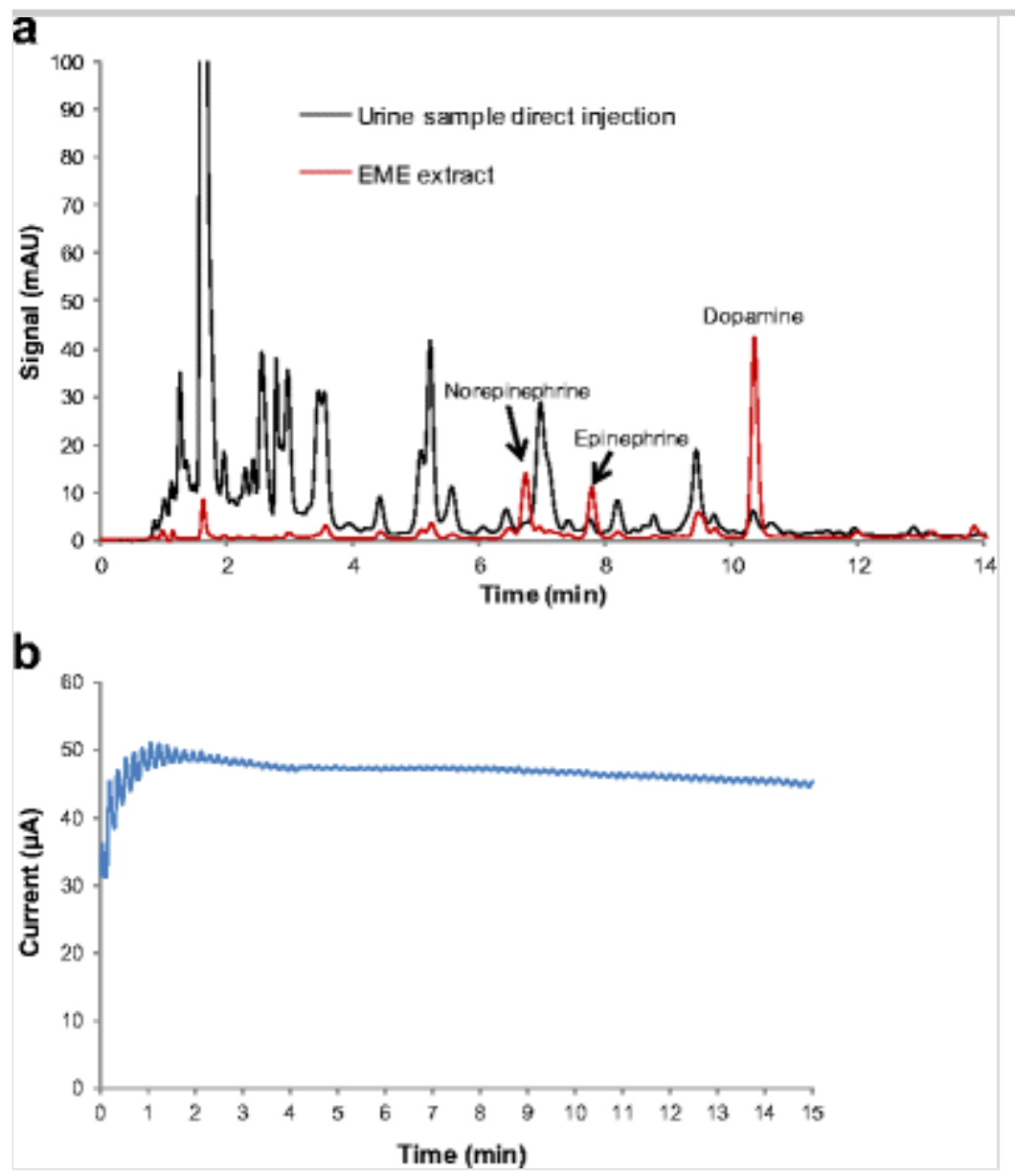

\section{Evaluation}

Finally, the complexation-mediated EME concept was combined with UHPLC-MS/MS and evaluated for quantification of $E$ and DA. The main purpose of this was to test if the new concept of complexation-mediated EME can provide reliable data. A complete validation was not considered at this stage. Quality analytical parameters were evaluated in pooled urine from three healthy volunteers. Standard addition calibration was used due to the matrix effects. To this end, pooled urine sample was diluted with $20 \mathrm{mM}$ phosphate buffer of $\mathrm{pH} 4$ (volume ratio 1:1) and calibration curves were constructed using standards of five concentration levels from 25 to $125 \mu \mathrm{g} \mathrm{L}^{-1}$. The content of $\mathrm{E}$ in the pooled urine sample was under the limit of detection (LOD) of the method whereas the content of DA was under the limit of quantification (LOQ). Correlation coefficient values $(r)$ were 0.990 for $\mathrm{E}$ and 0.996 for DA. The Student's $t$ test was applied to assess the linearity showing values of $11.91(r=0.990, N=5)$ for $\mathrm{E}$ and to $8.20(r=0.996, N=5)$ for DA, thus rejecting the null hypothesis of 
non-linear correlation for a $5 \%$ significance level and 3 degrees of freedom $\left(t_{0.05,3}=3.18\right)$ [29]. The repeatability of the method, expressed as coefficient of variation (CV), was determined by five consecutive extractions from diluted pooled urine sample spiked at a concentration level of $50 \mu \mathrm{g} \mathrm{L}^{-1}$. CV was $13 \%$ for both E and DA.

Extraction recoveries of the proposed procedure were found by the following strategy. First, diluted pooled urine sample was spiked at $50 \mu \mathrm{g} \mathrm{L}^{-1}$ with E and DA and subjected to EME. Then, EME was conducted from non-spiked diluted pooled urine and the final extract was spiked at $50 \mu \mathrm{g} \mathrm{L}^{-1}$. Signals obtained in both experiments were compared and, considering acceptor and sample solution volumes (i.e., $25 \mu \mathrm{L}$ and $1 \mathrm{~mL}$, respectively), extraction recoveries were calculated to $(5.5 \pm 0.9) \%$ for $\mathrm{E}$ and $(15 \pm 2) \%$ for DA $(n=5)$. At this recovery level, LODs $(\mathrm{S} / N=3)$ were 5.0 and $1.8 \mu \mathrm{g} \mathrm{L}^{-1}$, and LOQs $(\mathrm{S} / N=10)$ were 16.5 and $6.0 \mu \mathrm{g} \mathrm{L}^{-1}$ for E and DA, respectively. Enrichment factors were 2.2 for $E$ and 6.0 for DA. Although low enrichment factor were obtained, they could be further improved increasing sample and acceptor phases volume ratios.

Finally, diluted pooled urine sample was spiked at a known concentration level (i.e., $50 \mu \mathrm{g} \mathrm{L}^{-1}$ ) and analyzed by standard addition calibration using standards of five concentration levels from 25 to $125 \mu \mathrm{g} \mathrm{L}^{-1}$. Relative recoveries were calculated as ratio between found and spiked concentrations being $(91 \pm 26) \%$ for $\mathrm{E}$ and $(117 \pm 20) \%$ for DA, where standard deviation values were calculated using the $s_{\mathrm{XE}}$ (i.e., standard deviation of $x$-value estimated using regression line [29]).

\section{Conclusions}

In this work, complexation-mediated EME of highly polar basic drug substances was demonstrated for the first time using selected catecholamines as model analytes. Complexation in the bulk sample with water-soluble PBA derivatives added to the sample, and complexation at the sample/SLM interface with water-insoluble PBA derivatives added to the SLM were tested, and the latter concept appeared to be most efficient. Thus, complexation of the catecholamines with TFPBA at the sample/SLM interface was found to enhance the mass transfer across the SLM. Because the complexation reaction involved substances with two phenolic groups in ortho position only, the reaction was selective and therefore complexation- 
mediated EME appeared to be selective even from biological fluids. Thus, although the SLM permitted mass transfer of target analytes with $-1>\log$ $P>-2$, most bulk matrix components in human urine was unable to pass the SLM, and acceptable sample clean-up was achieved. Additionally, the current in the complexation-mediated EME system was easily controlled and kept below $50 \mu \mathrm{A}$, and therefore the system provided acceptable stability. The complexation-mediated EME concept was combined with UHPLC-MS/MS to develop a model application. Although the work presented in this paper is preliminary in nature, complexation-mediated EME showed potential and extraction of basic drugs with $\log \mathrm{P}$ in the range -1 to -2 was demonstrated for the first time. Complexation-mediated EME should be investigated in more detail in the future. With this concept, analyte detection may be performed with instruments much more simple than mass spectrometry (as used in this initial work), and this may open new and very interesting future possibilities.

\section{Acknowledgements}

The authors would like to thank the Spanish Ministry of Science and Innovation (project n. CTQ2011-23968) and Generalitat Valenciana (Spain) (projects n. GVA/2014/096 and PROMETEO/2013/038) for the financial support. E. Fernández thanks Spanish Ministry of Education for her FPU grant (FPU13/03125) and mobility grant (EST15/00074).

Compliance with ethical standards

Informed consent was obtained from all individual participants included in the study. Urine samples were collected from healthy volunteers and randomized. Collection was performed in accordance with ethical standards and approved by the Director of School of Pharmacy (University of Oslo, Norway).

Conflict of interest The authors declare that they have no conflicts of interest.

\section{Electronic supplementary material}

\section{ESM 1}

(PDF $171 \mathrm{~kb})$ 


\section{References}

1. Ghambarian M, Yamini Y, Esrafili A. Developments in hollow fiber based liquid-phase microextraction: principles and applications. Microchim Acta. 2012;177:271-94.

2. Huang C, Jensen H, Seip KF, Gjelstad A, Pedersen-Bjergaard S. Mass transfer in electromembrane extraction - the link between theory and experiments. J Sep Sci. 2016;39:188-97.

3. Fernández E, Vidal L. Liquid-phase microextraction techniques. In: Pena-Pereira F, editor. Miniaturization sample preparation. Warsaw: De Gruyter Open; 2014. p. 191-252.

4. Gjelstad A, Pedersen-Bjergaard S. Recent developments in electromembrane extraction. Anal Methods. 2013;5:4549-57.

5. Marothu VK, Gorrepati M, Vusa R. Electromembrane extraction-a novel extraction technique for pharmaceutical, chemical, clinical and environmental analysis. J Chromatogr Sci. 2013;51:619-31.

6. Gjelstad A, Rasmussen KE, Pedersen-Bjergaard S. Electrokinetic migration across artificial liquid membranes tuning the membrane chemistry to different types of drug substances. J Chromatogr A. 2006;1124:29-34.

7. Nojavan S, Fakhari AR. Electro membrane extraction combined with capillary electrophoresis for the determination of amlodipine enantiomers in biological samples. J Sep Sci. 2010;33:3231-8.

8. Fakhari AR, Tabani H, Nojavan S, Abedi H. Electromembrane extraction combined with cyclodextrin-modified capillary electrophoresis for the quantification of trimipramine enantiomers. Electrophoresis. 2012;33:506-15.

9. Davarani SSH, Najarian AM, Nojavan S, Tabatabaei M-A.

Electromembrane extraction combined with gas chromatography for quantification of tricyclic antidepressants in human body fluids. Anal Chim Acta. 2012;725:51-6. 
10. Hasheminasab KS, Fakhari AR. Development and application of carbon nanotubes assisted electromembrane extraction (CNTs/EME) for the determination of buprenorphine as a model of basic drugs from urine samples. Anal Chim Acta. 2013;767:75-80.

11. Ahmar H, Fakhari AR, Tabani H, Shahsavani A. Optimization of electromembrane extraction combined with differential pulse voltammetry using modified screen-printed electrode for the determination of sufentanil. Electrochim Acta. 2013;96:117-23.

12. Huang C, Eibak LEE, Gjelstad A, Shen X, Trones R, Jensen H, Pedersen-Bjergaard S. Development of a flat membrane based device for electromembrane extraction: a new approach for exhaustive extraction of basic drugs from human plasma. J Chromatogr A. 2014;1326:7-12.

\section{Asl YA, Yamini Y, Seidi S, Amanzadeh H. Dynamic} electromembrane extraction: automated movement of donor and acceptor phases to improve extraction efficiency. J Chromatogr A. 2015;1419:10-8.

14. Rouhollahi A, Kouchaki M, Seidi S. Electrically stimulated liquid phase microextraction combined with differential pulse voltammetry: a new and efficient design for in situ determination of clozapine from complicated matrices. RSC Adv. 2016;6:12943-52.

15. Gjelstad A, Rasmussen KE, Pedersen-Bjergaard S.

Electromembrane extraction of basic drugs from untreated human plasma and whole blood under physiological $\mathrm{pH}$ conditions. Anal Bioanal Chem. 2009;393:921-8.

16. Eibak LEE, Gjelstad A, Rasmussen KE, Pedersen-Bjergaard S. Kinetic electro membrane extraction under stagnant conditions-fast isolation of drugs from untreated human plasma. J Chromatogr A. 2010;1217:5050-6.

17. Jamt REG, Gjelstad A, Eibak LEE, Øiestad EL, Christophersen AS, Rasmussen KE, Pedersen-Bjergaard S. Electromembrane extraction of stimulating drugs from undiluted whole blood. J Chromatogr A. 
18. Šlampová A, Kubáň $\mathrm{P}$, Boček P. Electromembrane extraction using stabilized constant d.c. electric current-a simple tool for improvement of extraction performance. J Chromatogr A. 2012;1234:32-7.

19. Kjelsen IJØ, Gjelstad A, Rasmussen KE, Pedersen-Bjergaard S. Low-voltage electromembrane extraction of basic drugs from biological samples. J Chromatogr A. 2008;1180:1-9.

20. Seip KF, Faizi M, Vergel C, Gjelstad A, Pedersen-Bjergaard S. Stability and efficiency of supported liquid membranes in electromembrane extraction-a link to solvent properties. Anal Bioanal Chem. 2014;406:2151-61.

21. Huang C, Seip KF, Gjelstad A, Pedersen-Bjergaard S.

Electromembrane extraction for pharmaceutical and biomedical analysis_quo vadis. J Pharm Biomed Anal. 2015;113:97-107.

22. Huang C, Seip KF, Gjelstad A, Pedersen-Bjergaard S.

Electromembrane extraction of polar basic drugs from plasma with pure bis(2-ethylhexyl) phosphite as supported liquid membrane. Anal Chim Acta. 2016;934:80-7.

23. Bicker J, Fortuna A, Alves G, Falcão A. Liquid chromatographic methods for the quantification of catecholamines and their metabolites in several biological samples-a review. Anal Chim Acta. 2013;768:12-34.

24. Paugam M-F, Valencia LS, Boggess B, Smith BD. Selective dopamine transport using a crown boronic acid. J Am Chem Soc. 1994;116:11203-4.

25. Takeuchi M, Koumoto K, Goto M, Shinkai S. Efficient glucoside extraction mediated by a boronic acid with an intramolecular quaternary ammonium ion. Tetrahedron. 1996;52:12931-40.

26. Di Luccio M, Smith BD, Kida T, Borges CP, Alves TLM.

Separation of fructose from a mixture of sugars using supported liquid 
membranes. J Memb Sci. 2000;174:217-24.

27. Kanamori T, Funatsu T, Tsunoda M. Determination of catecholamines and related compounds in mouse urine using columnswitching HPLC. Analyst. 2016;141:2568-73.

28. Kubán P, Boček P. The effects of electrolysis on operational solutions in electromembrane extraction: the role of acceptor solution. $\mathrm{J}$ Chromatogr A. 2015;1398:11-9.

29. Miller JN, Miller JC. Statistics and chemometrics for analytical chemistry. fifth ed. London: Pearson Prentice Hall; 2005. 